\title{
Anesthetic management of a patient with a non-functioning right lung who underwent left lung resection.
}

Izumi Kawagoe, Masakazu Hayashida, Daizoh Satoh, Eiichi Inada.

Department of Anesthesiology and Pain Medicine, Juntendo University, Faculty of Medicine, Tokyo, Japan.
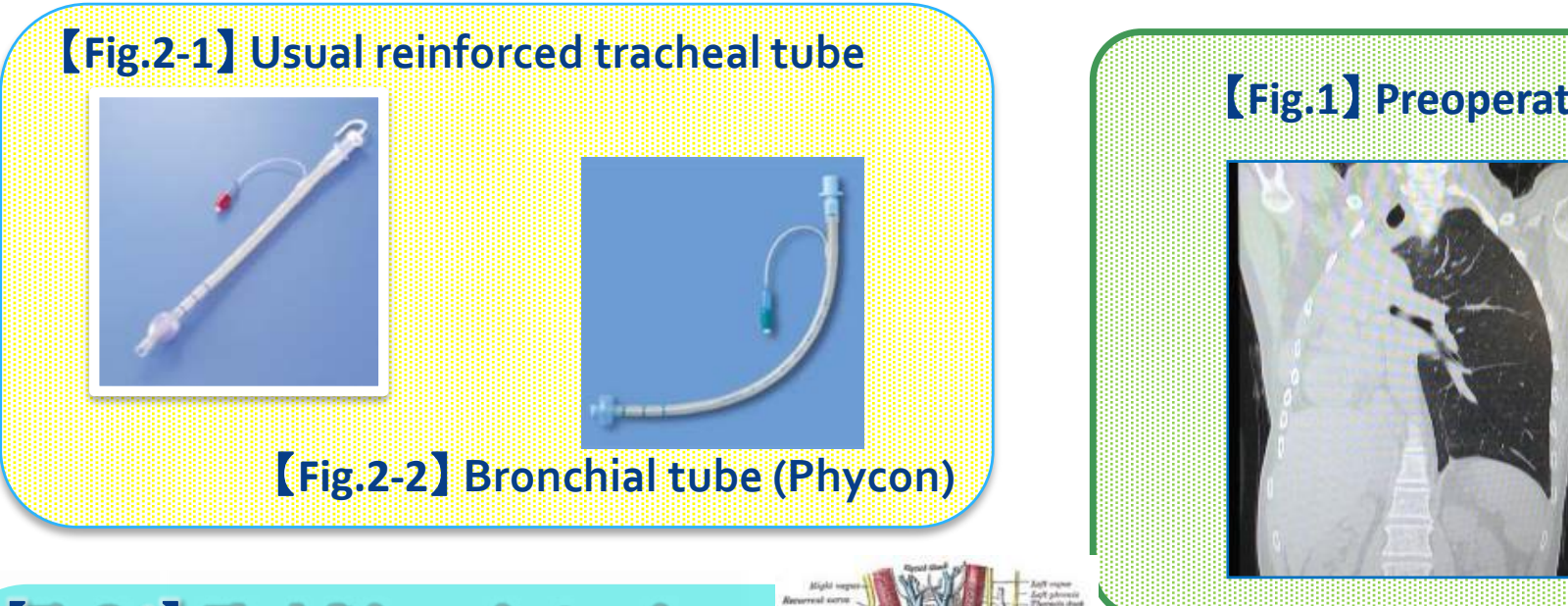

[Fig.1] Preoperative radiographic images
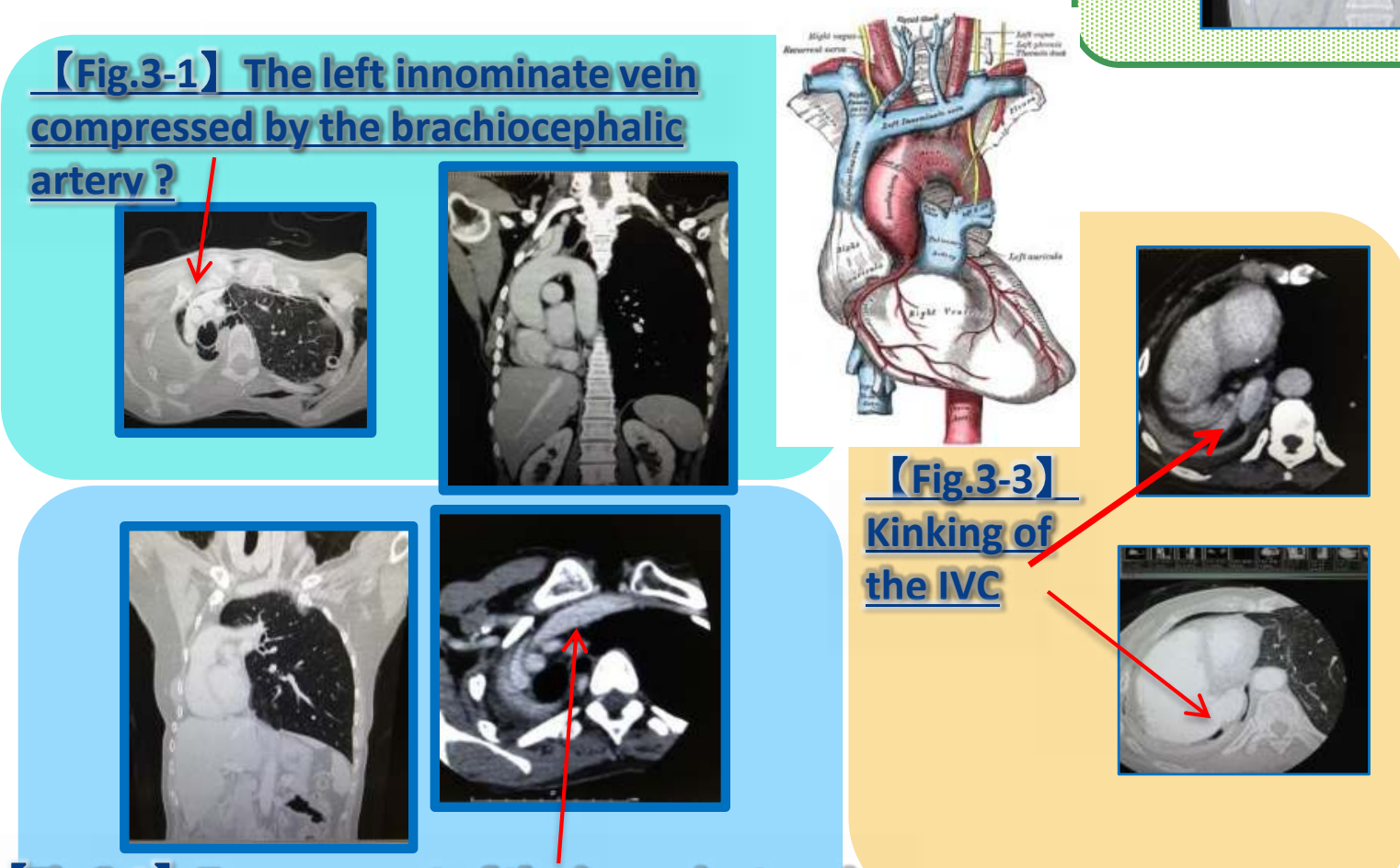

[Fig.3-2) Engorgement of the innominate vein

Background; Some long-term survivors after lung cancer surgery may require additional lung surgery for additional cancer. In the second surgery, special precautions are required regarding functional and anatomical changes of the remaining lungs. We experienced repeated episodes of sudden hypotension associated with brief apneic periods during left lung resection in a patient with a non-functioning right lung.

Case report; A 58-year-old woman $(154 \mathrm{~cm}, 41 \mathrm{~kg})$ was scheduled for a left superior segmentectomy for lung cancer. She had undergone a right upper lobectomy and superior segmentectomy 11 years before. Function of the residual right lung was abolished due to radiotherapy-induced pulmonary fibrosis. The mediastinal structures were located completely in the right thoracic cavity. Her major respiratory function data were TVC $=1.65 \mathrm{~L}(60.5 \%)$, $\mathrm{FEV} 1=1.38 \mathrm{~L}, \mathrm{PaCO} 2=44 \mathrm{mmHg}$, and $\mathrm{PaO} 2=67 \mathrm{mmHg}$. She was free from pulmonary hypertension. Anesthesia was induced and maintained with combined general and epidural anesthesia. After failure in an attempt to place an endobronchial tube in the left main bronchus under fiberscopic guidance for protection of the probably fragile right lung, we placed a wire-reinforced tracheal tube above the carina. Fortunately, the fibrotic right lung did not reexpand nor receive barotrauma due to positive pressure ventilation (PPV). Repeated cycles of intermittent apnea for 3-5 min and PPV were used to facilitate the segmentectomy. While brief apneic periods were repeated, sudden decreases in blood pressure, cardiac output, and $\mathrm{SpO} 2$ occurred repeatedly, which resolved with resumption of PPV and administration of phenylephrine $(0.1 \mathrm{mg} /$ time $)$. The segmentectomy could be completed without extreme hypercapnia or hypoxemia. She was extubated $10 \mathrm{~min}$ after the end of surgery. She discharged from hospital 10 days later.
【Fig.4】 Changes in cardiax output/index, Sp02, heart rate, and blood pressue
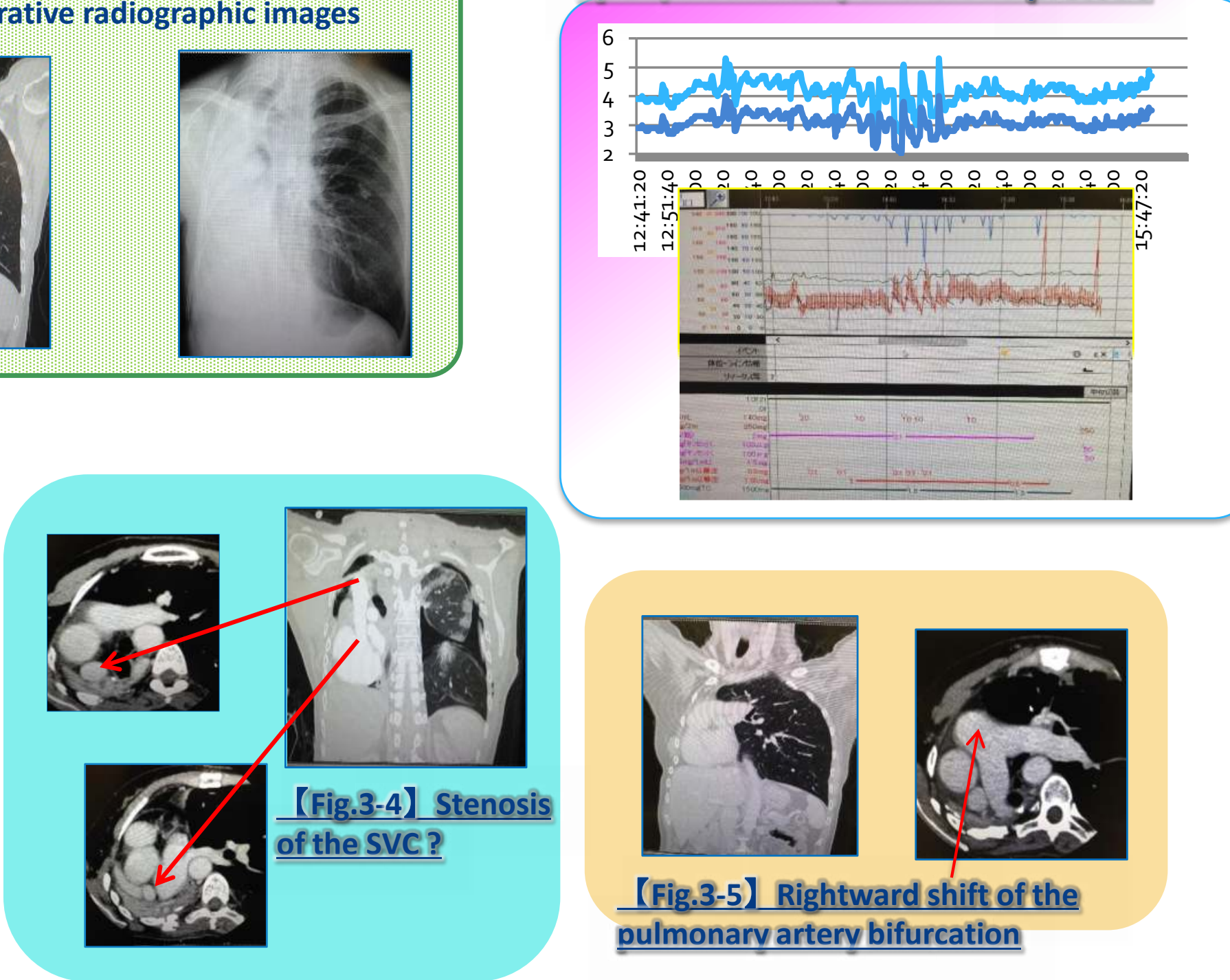

(Fig.3-5】 Rightward shift of the pulmonarvartervbifurcation

\begin{abstract}
Discussion and Learning points; Repeated hypotension during repeated brief apneic periods probably resulted from hypoxic pulmonary vasoconstriction, although SpO2 decreased only to $80 \%-90 \%$ during most apneic periods. Her right ventricle might not be able to eject a sufficient stroke volume against even a slightly vasoconstricted pulmonary arterioles possibly because of easy collapsibilty of her left lung and also because of remarkable anatomical deformity of the cardiovascular system, including the heart encaged in a narrow space in the right thoracic cavity, kinking and/or stenosis of major systemic veins, and an increased distance from the pulmonary artery bifurcation to left terminal pulmonary arterioles, as shown in Figures. Anesthesiologists must note anatomical changes of not only respiratory but also cardiovascular systems in patients scheduled for lung surgery following previous lung surgery.
\end{abstract}

\section{【Considerations of repeated hypotension during apneic periods】}

- Decreased pulmonary vascular beds in the decreased functioning lung volume + hypoxic pulmonary vasoconstriction during apnea in the easy-to-collapse left lung $\Rightarrow$ increased pulmonary vascular resistance $\Rightarrow$ decreased pulmonary blood flow $\Rightarrow$ decreased cardiac output

- Increased distance from the pulmonary artery bifurcation to the left terminal pulmonary arterioles $\Rightarrow$ increased right ventricular pressure required to drive up pulmonary blood flow against increased gravitational force in the patient in the right lateral position

- Confinement of the heart in the narrow cavity surrounded by irradiation-induced fibrotic/scarring tissues + kinking and/or stenosis of major systemic veins $\Rightarrow$ restricted venous return $\Rightarrow$ limited capacity of the right ventricle to increase its contractility against increased afterload using the Frank-Starling curve mechanism

\section{【Options of ventilation methods for post pneumonectomy patients with a sole functioning lung during additional lung surgery】}

1. Usual mechanical ventilation of the remaining lung with intermittent apnea to facilitate surgical procedures.

2. Selective partial lobar or segmental blockade with a blocker; difficulty in inserting a blocker in the presence of remarkable deformity of the tracheobronchial system.

3. Bronchial long reinforced tube; difficulty in inserting the tube in the presence of remarkable deformity.

4. Extra corporeal circulation; a potential option in case of severe hypoxemia, but with increased risk for perioperative hemorrhage and expected difficulty in usual arterial blood delivery and/or venous blood drainage due to the anatomical change in the intrathoracic cardiovascular system. 